\title{
WELL POSED REDUCED SYSTEMS FOR THE EINSTEIN EQUATIONS
}

\author{
YVONNE CHOQUET-BRUHAT \\ Gravitation et Cosmologie Relativiste \\ Université de Paris VI, t.22-12, 75252 Paris, France \\ E-mail: choquet@cicrp.jussieu.fr \\ JAMES W. YORK, JR. \\ Department of Physics and Astronomy \\ University of North Carolina \\ Chapel Hill, NC 27599-3255, U.S.A. \\ E-mail: york@physics.unc.edu
}

\begin{abstract}
We review some well posed formulations of the evolution part of the Cauchy problem of General Relativity that we have recently obtained. We include also a new first order symmetric hyperbolic system based directly on the Riemann tensor and the full Bianchi identities. It has only physical characteristics and matter sources can be included. It is completely equivalent to our other system with these properties.
\end{abstract}

1. Introduction. We will review some recently obtained well posed formulations of the evolution part of the Cauchy problem (see [9]) in General Relativity, considered as the time history of the two fundamental forms of the geometry of a spacelike hypersurface, its metric $\bar{g}$ and its extrinsic curvature $K$. On such an hypersurface, for instance an "initial" one, these two quadratic forms must satisfy four equations, called constraints. The constraints can be posed and solved as an elliptic system by known methods.

The proof of the existence of a causal evolution in local Sobolev spaces of $\bar{g}$ and $K$ into an Einsteinian spacetime does not however result directly from the equations giving the time derivatives of $\bar{g}$ and $K$ in terms of space derivatives of these quantities in a straightforward $3+1$ decomposition of the Ricci tensor of the spacetime metric, which contains also the lapse and shift characterizing the time lines. These equations do not

1991 Mathematics Subject Classification: Primary 35Q75; Secondary 83C05.

Research of the second author was supported by NSF grants PHY 94-13207 and PHY 9318152/ASC 93-18152 (ARPA supplemented).

The paper is in final form and no version of it will be published elsewhere. 
appear as a hyperbolic system for arbitrary lapse and shift, in spite of the fact that their characteristics are only the light cone and the time axis (see [13]).

In this paper, we review different methods that we have used recently for obtaining a hyperbolic evolution system for these geometrical unknowns with only physical characteristics which manifest their propagation governed by the light cone. Particularly interesting are first order symmetric hyperbolic systems, since they seem to be the most amenable to numerical computations. We construct explicitly such a system, using a harmonic time slicing condition. Its characteristics are the physical light cone and the time direction orthogonal to the space sub-manifolds of the chosen slicing. Among the propagated quantities is, in effect, the Riemann curvature. The space coordinates and the shift are arbitrary and, in this sense, the system is gauge invariant. In fact, we can make the lapse also take arbitrary values by introducing a given arbitrary function in its definition.

2. 3+1 Decomposition of the Riemann and Ricci tensors. We suppose in all this paper that the spacetime is a smooth manifold, $V=M \times \Re$, endowed with a metric $g$ of signature $(-,+,+,+)$, such that the "slices" are spacelike. This hypothesis is no restriction for globally hyperbolic spacetimes.

We choose on $V$ a moving coframe such that the dual frame has a time axis orthogonal to the slices $M_{t}$ while the space axes are tangent to them, namely we set

$$
\begin{array}{r}
\theta^{0}=d t \\
\theta^{i}=d x^{i}+\beta^{i} d t
\end{array}
$$

with $t \in \Re$ and $x^{i}, i=1,2,3$ local coordinates on $M$. The Pfaff derivatives $\partial_{\alpha}$ with respect to $\theta^{\alpha}$ are

In this coframe, the metric $g$ reads

$$
\begin{array}{r}
\partial_{0} \equiv \frac{\partial}{\partial t}-\beta^{i} \partial_{i} \\
\partial_{i} \equiv \frac{\partial}{\partial x^{i}} .
\end{array}
$$

$$
d s^{2}=g_{\alpha \beta} \theta^{\alpha} \theta^{\beta} \equiv-N^{2}\left(\theta^{0}\right)^{2}+g_{i j} \theta^{i} \theta^{j} .
$$

The $t$-dependent scalar $N$ and space vector $\beta$ are called the lapse and shift of the slicing. Any spacetime tensor decomposes into sets of time dependent space tensors by projections on the tangent space or the normal to $M_{t}$.

We define for any $t$-dependent space tensor $T$ another such tensor, $\hat{\partial}_{0} T$, of the same type by setting

$$
\hat{\partial}_{0} \equiv \frac{\partial}{\partial t}-\mathcal{L}_{\beta}
$$

where $\mathcal{L}_{\beta}$ is the Lie derivative on $M_{t}$ with respect to $\beta$.

The extrinsic curvature of the slices is the time dependent space tensor given by

$$
K_{i j} \equiv-\frac{1}{2} N^{-1} \hat{\partial}_{0} g_{i j}
$$


We denote by an overbar geometric quantities associated with the Riemannian metric $\bar{g} \equiv g_{i j} d x^{i} d x^{j}$ induced by $g$ on the slices.

The Riemann curvature tensor of $g$ admits the following decomposition into time dependent space tensors (cf. [18], zero shift; [6], arbitrary shift; [19], spacetime viewpoint)

$$
\begin{aligned}
& R_{i k l}^{j}=\bar{R}_{i k l}^{j}-K_{k}^{j} K_{l i}+K_{l}^{j} K_{k i} \\
& R_{i k l}{ }^{0}=N^{-1}\left(\bar{\nabla}_{k} K_{l i}-\bar{\nabla}_{l} K_{k i}\right) \\
& R_{i}{ }^{0}{ }_{j 0}=-\left(N^{-1} \hat{\partial}_{0} K_{i j}+K_{i m} K_{j}^{m}+N^{-1} \bar{\nabla}_{j} \partial_{i} N\right)
\end{aligned}
$$

From these formulas result the following ones for the Ricci curvature, where we have denoted by $H$ the mean extrinsic curvature of the space slices, $H=K_{i}^{i}$ :

$$
\begin{aligned}
R_{i j} & \equiv-N^{-1} \hat{\partial}_{0} K_{i j}+H K_{i j}-2 K_{i m} K_{j}^{m}-N^{-1} \bar{\nabla}_{j} \partial_{i} N+\bar{R}_{i j} \\
R_{0 j} & \equiv N\left(-\bar{\nabla}_{h} K_{j}^{h}+\partial_{j} H\right) \\
R_{00} & \equiv N\left(\bar{\nabla}^{i} \partial_{i} N-N K_{i j} K^{i j}+\partial_{0} H\right)
\end{aligned}
$$

From these identities results the following one for the Einstein tensor, leading to the so-called "Hamiltonian constraint":

$$
G_{00} \equiv R_{00}-\frac{1}{2} g_{00} R \equiv \frac{1}{2} N^{2}\left(\bar{R}-K . K+H^{2}\right)
$$

3. Wave equations for $K$. Local existence theorems. Using the relation between $\hat{\partial}_{0} g_{i j}$ and $K_{i j}$, we obtain (with no factor $\frac{1}{2}$ in the index symmetrization)

$$
\begin{aligned}
\hat{\partial}_{0} \bar{R}_{i j} \equiv & -\bar{\nabla}^{h} \bar{\nabla}_{(i}\left(N K_{j) h}\right)+\bar{\nabla}_{h} \bar{\nabla}^{h}\left(N K_{i j}\right)+\bar{\nabla}_{j} \partial_{i}(N H) \\
\equiv & -N \bar{\nabla}_{(i} \bar{\nabla}^{h} K_{j) h}-\bar{\nabla}^{h} \partial_{(i} N K_{j) h}-2 N \bar{R}_{i j m}^{h} K_{h}^{m}-N \bar{R}_{m(i} K_{j)}^{m}+ \\
& \bar{\nabla}_{h} \bar{\nabla}^{h}\left(N K_{i j}\right)+\bar{\nabla}_{j} \partial_{i}(N H) .
\end{aligned}
$$

We now use the expression for $R_{0 i}$ and $R_{i j}$ to obtain the identity

$$
\begin{aligned}
\Omega_{i j} \equiv & \hat{\partial}_{0} R_{i j}-\bar{\nabla}_{(i} R_{j) 0} \\
\equiv & -\hat{\partial}_{0}\left(N^{-1} \hat{\partial}_{0} K_{i j}\right)+\hat{\partial}_{0}\left(H K_{i j}-2 K_{i m} K_{j}^{m}\right)-\hat{\partial}_{0}\left(N^{-1} \bar{\nabla}_{j} \partial_{i} N\right)- \\
& N \bar{\nabla}_{i} \partial_{j} H-\bar{\nabla}_{(i}\left(K_{j) h} \partial^{h} N\right)-2 N \bar{R}_{i j m}^{h} K_{h}^{m}-N \bar{R}_{m(i} K_{j)}^{m}+ \\
& \bar{\nabla}_{h} \bar{\nabla}^{h}\left(N K_{i j}\right)+H \bar{\nabla}_{j} \partial_{i} N
\end{aligned}
$$

This identity shows that for a solution of the Einstein equations

$$
R_{\alpha \beta}=\rho_{\alpha \beta}
$$

the extrinsic curvature $K$ satisfies a second order differential system which is quasi diagonal with the principal part the wave operator, except for the terms $\bar{\nabla}_{i} \partial_{j} H$. The other unknowns $\bar{g}$ and $N$ appear at second order except for the term $\hat{\partial}_{0} \bar{\nabla}_{j} \partial_{i} N$. These facts have been used in two different ways to obtain for $K$ a quasi diagonal, wave type system.

1. Harmonic time slicing. One eliminates at the same time the third derivatives of $N$ and the second derivatives of $H$ (cf. in the case of zero shift [8] and for arbitrary shift [10]) by requiring $N$ to satisfy

$$
\partial_{0} N+N^{2} H=0
$$


The second order equation for $K$ reads then as a wave type nonlinear system:

$$
N \square K_{i j}=N Q_{i j}+\Theta_{i j}
$$

where we set

$$
\begin{aligned}
\square K_{i j} \equiv & -N^{-2} \hat{\partial}_{0} \hat{\partial}_{0} K_{i j}+\bar{\nabla}^{h} \bar{\nabla}_{h} K_{i j} \\
N Q_{i j} \equiv & -K_{i j} \partial_{0} H+2 g^{h m} K_{m(i} \hat{\partial}_{0} K_{j) h}+4 N g^{h l} g^{m k} K_{l k} K_{i m} K_{j h}+ \\
& \left(2 \bar{\nabla}_{(i} K_{j) l}\right) \partial^{l} N-2 H N^{-1} \partial_{i} N \partial_{j} N-2 \partial_{(i} N \partial_{j)} H- \\
& 3 \partial_{h} N \bar{\nabla}^{h} K_{i j}-K_{i j} \bar{\nabla}^{h} \bar{\nabla}_{h} N-N^{-1} K_{i j} \partial^{h} N \partial_{h} N+ \\
& N^{-1} K_{h(i} \partial_{j)} N \partial^{h} N+\left(\bar{\nabla}_{(i} \partial^{h} N\right) K_{j) h}+2 N \bar{R}_{i j m}^{h} K_{h}^{m}+ \\
& N \bar{R}_{m(i} K_{j)}^{m}-2 H \bar{\nabla}_{j} \partial_{i} N \\
\Theta_{i j} \equiv & \hat{\partial}_{0} \rho_{i j}-\bar{\nabla}_{(i} \rho_{j) 0}
\end{aligned}
$$

The equation $\left(N^{\prime}\right)$ expresses that the time coordinate is harmonic.

Using the expression for $H$ we see that $\left(N^{\prime}\right)$ reads

$$
\hat{\partial}_{0} \log \left(N /(\operatorname{det} \bar{g})^{\frac{1}{2}}\right)=0
$$

The general solution of this equation is

$$
N=\alpha^{-1}\left(\operatorname{det} \bar{g}^{\frac{1}{2}}\right)
$$

where $\alpha$ is an arbitrary tensor density such that

$$
\hat{\partial}_{0} \alpha=0 .
$$

The algebraic expression of the harmonic time-slicing condition is called "algebraic gauge."

If we replace $N$ by the value obtained above in the second order equation for $K$ we obtain a quasi diagonal system with principal part the wave operator, with terms depending on $\bar{g}$ and its derivatives of order $\leq 2$. This system reduces to a third order hyperbolic system when we replace $K$ by $-(2 N)^{-1} \hat{\partial}_{0} \bar{g}$. The local existence theorem of Leray for the solution of hyperbolic systems gives immediately the local in time existence of solutions of this reduced system, in local in space Sobolev spaces, with the smallest index known for generic solutions of Einstein's equations, and domain of dependence determined by the light cone. It can be proved (see [2]) that a solution of the reduced system on $M \times I$ is a solution, in algebraic gauge, of the full Einstein equations if the initial data satisfy the Einstein equations on $M_{0}$.

R e mark. If we take for $\alpha$ an arbitrary given function we still obtain a quasi diagonal system for $K$, with additional a priori given terms. Such a generalization is equivalent to replacing $\left(N^{\prime}\right)$ by the equation

$$
N^{-1} \partial_{0} N+N H=f
$$

with $f$ an arbitrary function. (The algebraic gauge was introduced in [8]. It is used in [13]. A generalization was introduced in [14]. The wave operator form needed to produce the first order symmetric hyperbolic causal system given below was introduced in [10].) 
2. Mean curvature gauge. Replace in the term $\bar{\nabla}_{j} \partial_{i} H$ the mean curvature $H$ by an a priori given function $h$ (a procedure used in [12] with $h=0$, in the asymptotically euclidean case). The equations $\Omega_{i j}=\Theta_{i j}$ become then, when $N$ is known, a quasi diagonal second order system for $K$ with principal part the wave operator, namely:

$$
\square K_{i j}=P_{i j}+\Theta_{i j}
$$

where

$$
\square K_{i j} \equiv-\hat{\partial}_{0} \hat{\partial}_{0}\left(N^{-1} K_{i j}\right)+\bar{\nabla}^{h} \bar{\nabla}_{h}\left(N K_{i j}\right)
$$

Here, $P_{i j}$ depends only on $K$ and its first derivatives, on $\bar{g}, N$ and $\partial_{0} N$ together with their space derivatives of order $\leq 2$. It is given by

$$
\begin{aligned}
P_{i j} \equiv & \hat{\partial}_{0}\left(-H K_{i j}+2 g^{h m} K_{(i m} K_{j)}^{m}\right)+\hat{\partial}_{0}\left(N^{-1} \bar{\nabla}_{j} \partial_{i} N\right)+\bar{\nabla}^{h}\left(\partial_{(i} N K_{j) h}\right)+ \\
& 2 N \bar{R}_{i j m}^{h} K_{h}^{m}+N \bar{R}_{m(i} K_{j)}^{m}-H \bar{\nabla}_{j} \partial_{i} N+N \bar{\nabla}_{j} \partial_{i} h,
\end{aligned}
$$

while $\Theta_{i j}$, zero in vacuum, is

$$
\Theta_{i j} \equiv \hat{\partial}_{0} \rho_{i j}-\bar{\nabla}_{(i} \rho_{j) 0} .
$$

When $\beta, N$ and the sources $\rho$ are known the above equation together with

$$
\hat{\partial}_{0} g_{i j}=-2 N K_{i j}
$$

are again a third order quasi diagonal system for $\bar{g}$, hyperbolic if $N>0$ and $\bar{g}$ is properly Riemannian.

The condition $H=h$ is a "mean curvature" gauge choice which imposes, through the equation $R_{0}^{0}=\rho_{0}^{0}$, that $N$ satisfy the following elliptic equation on each slice

$$
\bar{\nabla}^{i} \partial_{i} N-\left(K_{i j} K^{i j}-\rho_{0}^{0}\right) N=-\partial_{0} h
$$

Note that for energy sources satisfying the strong energy condition we have $-\rho_{0}^{0} \geq 0$, as well as $K . K \equiv K_{i j} K^{i j} \geq 0$, an important property for the solution of the elliptic equation. In the given mean curvature gauge the unknowns $N$ and $\bar{g}$ satisfy, for any choice of the shift $\beta$, a mixed elliptic and hyperbolic system.

Local existence theorems (i.e. in a neighborhood of $M$ in $M \times \Re$ ) have been proven (see [10], [11]) when $M$ is compact and when $M$ is asymptotically euclidean.

\subsection{Case of compact $M$}

THEOREM 1. Let $(M, e)$ be a smooth compact Riemannian manifold. Let there be given on $M \times I$, with $I \equiv[0, T]$, a "pure space" smooth vector field $\beta$ and a function $h$ such that

$$
h \in \bigcap_{2 \leq k \leq 3} C^{3-k}\left(I, H_{k}\right), \quad \partial_{0} h \geq 0, \quad \partial_{0} h \not \equiv 0 .
$$

There exists an interval $J \equiv[0, \ell], \ell \leq T$ such that the system $\left(K^{\prime \prime}\right),\left(g^{\prime}\right),(N)$ has one and only one solution on $M \times J$

$$
\begin{gathered}
\bar{g} \in \bigcap_{1 \leq k \leq 3} C^{3-k}\left(J, H_{k}\right) \\
K \in \bigcap_{1 \leq k \leq 2} C^{2-k}\left(J, H_{k}\right)
\end{gathered}
$$




$$
N \in \bigcap_{0 \leq k \leq 2} C^{2-k}\left(J, H_{2+k}\right)
$$

with $N>0$ and $\bar{g}$ uniformly equivalent to $e$, taking the initial data

$$
\begin{gathered}
g_{i j}(0, .)=\gamma_{i j} \in H_{3} \\
K_{i j}(0, .)=k_{i j} \in H_{2}
\end{gathered}
$$

if $\gamma$ is a properly riemannian metric uniformly equivalent to $e$ and $k . k \not \equiv 0$.

3.2. Case of $(M, e)$ euclidean at infinity. The manifold $M$ is the union of a compact set and a finite number of disjoint sets (its "ends") diffeomorphic to the exterior of a ball in $\Re^{3}$; the smooth given metric $e$ reduces on each end to the euclidean metric. The weighted Sobolev space $H_{s, \delta}$ on $(M, e)$ is the completion of $C_{0}^{\infty}$ in the norm

$$
\|f\|_{H_{s, \delta}} \equiv\left(\int_{M} \sum_{0 \leq k \leq s} \sigma^{2 k+2 \delta}\left|D^{k} f\right|^{2} \mu(e)\right)^{\frac{1}{2}}
$$

where $\sigma^{2} \equiv 1+d^{2}$ and $d$ is the distance in the metric $e$ to some fixed point in $M$.

The use of the theory of elliptic equations on an asymptotically euclidean manifold in $H_{s, \delta}$ spaces (see [7]) and of weighted energy estimates leads to the following theorem:

Theorem 2. The system $\left(K^{\prime \prime}\right),\left(g^{\prime}\right),(N)$ with Cauchy data $\gamma$ and $k$ on the manifold $(M, e)$ euclidean at infinity with given $\beta$ and $h$ on $M \times[0, T]$ has one and only one solution $(\bar{g}, K, N)$ on $M \times J, J \equiv[0, \ell]$ a sufficiently small subinterval of $I$, if the Cauchy data are such that $\gamma-e \in H_{3,-1}$ and is uniformly equivalent to e while $k \in H_{2,0}$ and $\dot{k} \in H_{1,1}$. The solution belongs on each $M_{t}$ to the same functional spaces as the data. It is such that $N>0$ and $\bar{g}$ is uniformly equivalent to $\gamma$.

Remark. The estimates show in fact that $\bar{g}-\gamma$ is such that for each $t$ we have:

$$
\bar{g}_{t}-\gamma \in H_{3,0}
$$

which is a stronger asymptotic fall off than $\gamma-e$ or $\bar{g}_{t}-e$ : this property is related to the Arnowitt-Deser-Misner theorem of mass conservation.

4. First order system (vacuum). We use the wave equation satisfied by $K$ to show (see [10], [1]) that in spacetime dimension 4 a solution of the vacuum Einstein equations, together with the harmonic time gauge condition, satisfies a first order symmetric system, hyperbolic if $\bar{g}$ is properly Riemannian and $N>0$. Such a system could be useful to establish a priori estimates relevant to global problems. It may be important for numerical computations (see [5]) because symmetric hyperbolic systems occur in many areas of mathematical physics, in particular in fluid dynamics, and effective codes have been developed to study such systems.

We have obtained for the unknowns $\bar{g}, K, N$ the equations

$$
\begin{aligned}
& \hat{\partial}_{0} g_{i j}=-2 N K_{i j} \\
& \partial_{0} N=-N^{2} H \\
& \square K_{i j} \equiv Q_{i j}
\end{aligned}
$$


To obtain a first order system we take as additional unknowns:

$$
\begin{array}{r}
N^{-1} \hat{\partial}_{0} K_{i j}=L_{i j} \\
\bar{\nabla}_{h} K_{i j}=M_{h i j} \\
\partial_{i} \log N=a_{i} \\
N^{-1} \hat{\partial}_{0} \partial_{i} \log N=a_{0 i} \\
\bar{\nabla}_{j} \partial_{i} \log N=a_{j i}
\end{array}
$$

We take as equation $\left(3^{\prime}\right)$

$$
\hat{\partial}_{0} K_{i j}=N L_{i j}
$$

The equation (3) gives

$$
\hat{\partial}_{0} L_{i j}-N \bar{\nabla}^{h} M_{h i j}=N\left(H L_{i j}-Q_{i j}\right)
$$

In three space dimensions the Riemann tensor is a linear function of the Ricci tensor:

$$
\bar{R}_{l i j m} \equiv g_{l j} \bar{R}_{i m}+g_{i m} \bar{R}_{j l}-g_{i j} \bar{R}_{l m}-g_{l m} \bar{R}_{i j}-\frac{1}{2}\left(g_{l j} g_{i m}-g_{i j} g_{l m}\right) \bar{R}
$$

Using the equation $R_{i j}=0$ to express $\bar{R}_{i j}$, we write $Q_{i j}$ as a polynomial in the unknowns and $g^{i j}$.

By using the identity

$$
\hat{\partial}_{0} \bar{\Gamma}_{i j}^{h} \equiv \bar{\nabla}^{h}\left(N K_{i j}\right)-\bar{\nabla}_{(i}\left(N K_{j)}^{h}\right)
$$

we see that for an arbitrary covariant vector $u_{i}$ we have

$$
\hat{\partial}_{0} \bar{\nabla}_{h} u_{i} \equiv \bar{\nabla}_{h} \hat{\partial}_{0} u_{i}+u_{l}\left(\bar{\nabla}_{h}\left(N K_{i}^{l}\right)-\bar{\nabla}_{i}\left(N K_{h}^{l}\right)-\bar{\nabla}^{l}\left(N K_{i h}\right)\right)
$$

From an analogous formula for tensors we deduce the equation

$$
\begin{aligned}
\hat{\partial}_{0} M_{h i j}-N \bar{\nabla}_{h} L_{i j}= & N\left[a_{h} L_{i j}+\left(M_{h(i}^{l} K_{j) l}+K_{l(i} M_{j) h}^{l}-K_{l(j} M_{i) h}^{l}\right)\right. \\
& \left.+K_{l(j}\left(K_{i)}^{l} a_{h}+a_{i)} K_{h}^{l}-a^{l} K_{i) h}\right)\right]
\end{aligned}
$$

On the other hand (2) implies

$$
\hat{\partial}_{0} a_{i}=N a_{0 i}=-N\left(M_{i h}^{h}+H a_{i}\right)
$$

while $a_{h i}$ and $a_{0 i}$ must satisfy

(7) $\hat{\partial}_{0} a_{h i}-N \bar{\nabla}_{h} a_{0 i}=N\left(a_{l}\left(M_{h i}{ }^{l}+M_{i h}{ }^{l}-M^{l}{ }_{i h}\right)+a_{l}\left(K_{i}^{l} a_{h}+K_{h}^{l} a_{i}-a^{l} K_{i h}\right)+a_{h} a_{0 i}\right)$

The condition (2) together with the Einstein equation $R_{0}^{0}$ imply that $N$ satisfies the inhomogeneous wave equation (this step brings in the Hamiltonian constraint determined by $\left.G_{00}\right)$

$$
\partial_{0} \partial_{0} N-N^{2} \bar{\nabla}^{h} \bar{\nabla}_{h} N=-N^{3} K_{i j} K^{i j}+2 N^{3} H^{2}
$$

Hence by differentiation, use of the Ricci formula, the definitions of $a_{h i}$ and $a_{0 i}$ and simplification through the use of $R_{0}^{0}=0$

$$
\begin{aligned}
\hat{\partial}_{0} a_{0 i}-N \bar{\nabla}^{h} a_{h i}= & N\left(2 a^{h} a_{i h}-2 K_{h l} M_{i}{ }^{h l}+H M_{i h}{ }^{h}-\bar{R}_{i}^{h} a_{h}\right) \\
& +N a_{i}\left(H^{2}+2 a_{h}^{h}+2 a_{h} a^{h}-2 K_{h j} K^{h j}\right)
\end{aligned}
$$

We use again the equation $R_{i j}=0$ to replace $\bar{R}_{i j}$ by its value in terms of the unknowns. We have obtained a first order system $\left(1,2,3^{\prime}, 4,5,6,7,8\right)$ in all the unknowns. The 
right hand sides are polynomial in the unknowns and $g^{i j}$; they do not depend on their derivatives. The left hand sides are linear operators on all the unknowns. Their coefficients depend on these unknowns, and not on their derivatives except for the derivatives of $\bar{g}$ which appear through the Christoffel symbols $\bar{\Gamma}_{i j}^{h}$. We can use the identity given above to write their evolution equation

$$
\hat{\partial}_{0} \bar{\Gamma}_{i j}^{h}=N\left(M_{i j}^{h}-M_{(i j)}^{h}-a^{h} K_{i j}-a_{(i} K_{j)}^{h}\right) .
$$

However, if we wish to display an explicitly covariant quasi linear first order system for all the unknowns, in particular if $M$ is not diffeomorphic to $\Re^{3}$, we introduce on $M$ an a priori given connection $E$, possibly derived from a metric $e$ which will also be used to define the Sobolev spaces on $M$. The derivatives in the connection of $\bar{g}$ and the given connection differ by terms linear in a tensor $S$, the difference of the two connections. The evolution of this tensor is given by

$$
\hat{\partial}_{0} S_{i j}^{h}=N\left(M_{i j}^{h}-M_{(i j)}^{h}-a^{h} K_{i j}-a_{(i} K_{j)}^{h}\right)-\hat{\partial}_{0} E_{i j}^{h}
$$

We have now obtained a quasi linear first order system covariant for all the unknowns. Its characteristic matrix, obtained by replacing in the principal matrix the operator $\partial$ by a covariant vector $\xi$, consists of blocks around the diagonal, some reduced to one element $\xi_{0}$ and some $4 \times 4$ matrices with determinant $\xi_{0}^{2}\left(\xi_{0}^{2}-N^{2} \xi^{i} \xi_{i}\right)$. The characteristics are the light cone and the axis orthogonal to the time slices. On the other hand, the system can be symmetrized by multiplication by a matrix consisting of blocks around the diagonal equal to one element, 1 , or the matrix $\left(g^{i j}\right)$. In other words, the system is a first order symmetrizable hyperbolic system, with domain of dependence determined by the light cone. Known local existence theorems apply to such a system.

5. Bianchi equations. Instead of taking as new unknowns the first derivatives of $K$ to obtain a first order symmetric hyperbolic system, one can use the Riemann tensor of the spacetime metric, which is linear in these derivatives, to rewrite the system in Section 4. It satisfies the Bianchi identities:

$$
\nabla_{\alpha} R_{\beta \gamma, \lambda \mu}+\nabla_{\beta} R_{\gamma \alpha, \lambda \mu}+\nabla_{\gamma} R_{\alpha \beta, \lambda \mu} \equiv 0
$$

These identities imply by contraction and use of the symmetries of the Riemann tensor

$$
\nabla_{\alpha} R_{\mu, \beta \gamma}^{\alpha}+\nabla_{\gamma} R_{\beta \mu}+\nabla_{\beta} R_{\gamma \mu} \equiv 0
$$

If the Ricci tensor $R_{\alpha \beta}$ satisfies the Einstein equations

$$
R_{\alpha \beta}=\rho_{\alpha \beta}
$$

then the previous identities imply the equations

$$
\nabla_{\alpha} R_{\mu, \beta \gamma}^{\alpha}=\nabla_{\beta} \rho_{\gamma \mu}-\nabla_{\gamma} \rho_{\beta \mu}
$$

The first equations with $(\alpha \beta \gamma)=(i j k)$ and the last one with $\mu=0$ do not contain derivatives of the Riemann tensor transversal to $M_{t}$; they are considered as constraints.

In a particular case (see [20]) of the general system considered below, the conservation of an initially vanishing Riemann tensor in vacuum was obtained. That result does not require analyticity when it is written in symmetric hyperbolic form as below. 
We wish first to show that the remaining equations are, for $n=3$ in the vacuum case, when $g$ is given, a symmetric first order hyperbolic system for the double two-form $R_{\alpha \beta, \lambda \mu}$. For this purpose, following Bel (see [4]) we introduce two pairs of "electric" and "magnetic" space tensors associated with a spacetime double two-form $A$,

$$
\begin{array}{r}
N^{2} E_{i j} \equiv A_{0 i, 0 j} \\
D_{i j} \equiv \frac{1}{4} \eta_{i h k} \eta_{j l m} A^{h k, l m} \\
N H_{i j} \equiv \frac{1}{2} \eta_{i h k} A_{, 0 j}^{h k} \\
N B_{j i} \equiv \frac{1}{2} \eta_{i h k} A_{0 j,}{ }^{h k}
\end{array}
$$

where $\eta_{i j k}$ is the volume form of $\bar{g}$. It results from the symmetry of the Riemann tensor $R$ with respect to its first and second pairs of indices ( $R$ is a "symmetric double two-form") that if $A \equiv R$, then $E$ and $D$ are symmetric while $H_{i j}=B_{j i}$. The Lanczos identity (see [15]) for a symmetric double two form like $R$, with a tilde representing the spacetime double dual, is

$$
\tilde{R}_{\alpha \beta, \lambda \mu}+R_{\alpha \beta, \lambda \mu}=C_{\alpha \lambda} g_{\beta \mu}-C_{\alpha \mu} g_{\beta \lambda}+C_{\beta \mu} g_{\alpha \lambda}-C_{\beta \lambda} g_{\alpha \mu}
$$

where $C_{\alpha \beta}=R_{\alpha \beta}-\frac{1}{4} g_{\alpha \beta} R$. It follows that when $R_{\alpha \beta}=\lambda g_{\alpha \beta}$, then $E=-D$ and $H=B$. In order to avoid introducing unphysical characteristics, and to be able to extend the treatment to the non vacuum case, we do not use these properties in the evolution equations, but write them as a first order system for an arbitrary double 2-form $A$ as follows

$$
\begin{aligned}
& \nabla_{0} A_{h k, 0 j}+\nabla_{k} A_{0 h, 0 j}-\nabla_{h} A_{0 k, 0 j}=0 \\
& \nabla_{0} A^{0}{ }_{i, 0 j}+\nabla_{h} A^{h}{ }_{i, 0 j}=\nabla_{0} \rho_{j i}-\nabla \rho_{0 i}
\end{aligned}
$$

and analogous equations with the pair $(0 \mathrm{j})$ replaced by $(\mathrm{lm})$. One obtains a first order system for the unknowns $E, H, D, B$ by using the following relations deduced from the definition of these tensors

$$
\begin{array}{r}
A_{h k, 0 j} \equiv N \eta_{h k}^{i} H_{i j} \\
A_{h k, l m} \equiv \eta_{h k}^{i} \eta_{l m}^{j} D_{i j} \\
A_{0 j, h k}=N \eta_{h k}^{i} B_{j i}
\end{array}
$$

The system obtained has a principal matrix consisting of 6 identical 6 by 6 blocks around the diagonal, which are symmetrizable and hyperbolic. Hence, the system is symmetric hyperbolic, when $g$ is a given metric such that $\bar{g}$ is properly Riemannian and $N>0$.

To relate the Riemann tensor to the metric $g$ we use the definition

$$
\hat{\partial}_{0} g_{i j}=-2 N K_{i j}
$$

and we use the $3+1$ identities given previously.

We consider two possibilities: each introduces a choice of gauge for $\mathrm{N}$.

1. We fix the mean curvature of the space slices, i.e. we set

$$
H=h
$$


with $h$ being a given function. We deduce from the identity giving $R_{i k l}^{0}$ the "elliptic" identity

$$
\bar{\nabla}^{h} \bar{\nabla}_{h} K_{i j}-\bar{R}_{j}^{h} K_{h i}-\bar{R}_{h i j m} K^{h m} \equiv \bar{\nabla}^{h}\left(N R_{i, j h}^{0}\right)-\bar{\nabla}_{j}\left(N^{-1} R_{0 i}\right)+\bar{\nabla}_{i} \partial_{j} H .
$$

We write an elliptic system linking the symmetric tensor $K$ with a double 2-form $A$ when $H=h$ by symmetrization of the above identity, replacement of $R$ by $A, H$ by $h$, and the spacetime Ricci tensor by $\rho$, zero in vacuum. Namely, we consider the system

$$
\bar{\nabla}^{h} \bar{\nabla}_{h} K_{i j}-\bar{R}_{(i}^{h} K_{j) h}-\bar{R}_{h i j m} K^{h m}=\bar{\nabla}^{h}\left(N A_{(i, j) h}^{0}\right)-\bar{\nabla}_{(i}\left(N^{-1} \rho_{j) o}\right)+\bar{\nabla}_{i} \partial_{j} h .
$$

This system is to be solved globally on each slice $M_{t}$, like the equation for $N$ in mean curvature gauge. We have obtained a mixed elliptic-hyperbolic system for the unknowns $\bar{g}, N, K, E, D, H, B$. It can be proved that this system is well posed in the case of compact or asymptotically euclidean $M$, as long as the elliptic operators are injective. This property holds for metrics $\bar{g}$ in a neighborhood of a metric $\gamma$ for which it holds, for example if $M \equiv \Re^{3}$ and $\gamma$ is the euclidean metric, or if $M \equiv S^{3}$ and $\gamma$ is the canonical metric of $S^{3}$.

2. We choose the algebraic form of the harmonic time-slicing condition, that is we set $N \equiv \alpha^{-1}|\operatorname{det} \bar{g}|^{1 / 2}$, with $\alpha$ a given tensor density. We follow the ideas used by Friedrich (see [13]) for the Weyl tensor to write a symmetric hyperbolic system for $K$ and $\bar{\Gamma}$, namely we consider the following identities, deduced from the definition of $K$ and the $3+1$ decomposition of the Riemann tensor:

$$
\hat{\partial}_{0} \bar{\Gamma}_{i j}^{h}+N \bar{\nabla}^{h} K_{i j}=K_{i j} \partial^{h} N-K_{(i}^{h} \partial_{j)} N-R_{0(i, j)}^{h}
$$

and

$$
\hat{\partial}_{0} K_{i j}+N \bar{R}_{i j}+\bar{\nabla}_{j} \partial_{i} N \equiv-2 N R_{i, 0 j}^{0}-N H K_{i j}+N R_{i j} .
$$

We obtain equations relating $\bar{\Gamma}$ and $K$, for a given double 2-form A, by replacing in these identities the Riemann tensor by $A$ and the Ricci tensor of spacetime by a given tensor $\rho$, zero in vacuum. To deduce from this system a symmetric hyperbolic first order system, with the algebraic form of the harmonic gauge, one uses the fact that in this gauge one has

$$
\bar{\Gamma}_{i h}^{h}=\partial_{i} \log N+\partial_{i} \log \alpha
$$

The second set of identities leads then to the following equations:

$$
\begin{aligned}
\hat{\partial}_{0} K_{i j}+N \partial_{h} \bar{\Gamma}_{i j}^{h}= & N\left(\bar{\Gamma}_{i h}^{m} \bar{\Gamma}_{j m}^{h}-\left(\bar{\Gamma}_{i h}^{h}-\partial_{i} \log \alpha\right)\left(\bar{\Gamma}_{j l}^{l}-\partial_{j} \log \alpha\right)\right)+ \\
& N \bar{\nabla}_{j} \partial_{i} \log \alpha-2 N A_{i, 0 j}^{0}-N H K_{i j}+N \rho_{i j}
\end{aligned}
$$

The system obtained for $K$ and $\bar{\Gamma}$ has a characteristic matrix composed of 6 blocks around the diagonal, each block a 4 by 4 matrix that is symmetrizable hyperbolic, with characteristic polynomial $\xi_{0}^{2} \xi^{\alpha} \xi_{\alpha}$.

The whole system for $A, K, \bar{\Gamma}, \bar{g}$ is symmetrizable hyperbolic, with characteristics the light cone and the normal to $M_{t}$.

Remark. It is somewhat involved to prove that a solution of the constructed system satisfies the Einstein equations if the initial data, $\gamma, k$, satisfy the constraints, but we can argue as follows. We consider the vacuum case with initial data $\gamma$ and $k$ satisfying the 
Einstein constraints. These initial data determine the initial values of $\bar{\Gamma}$, and also, if $\beta$ and $N$ are known, the initial values of $A_{i j, h m}, A_{j h, i 0}, A_{i 0, j h}$ by using the decomposition formulas. (We set $A$ equal to the Riemann tensor on the initial surface.) We use the Lanczos formula to determine $A_{i 0, j 0}$ initially. We know that our symmetrizable hyperbolic system has one and only one solution. Since a solution of Einstein's equations in algebraic gauge, proved to exist in a preceding section, satisfies together with its Riemann tensor the present system and takes the same initial values, that solution coincides with the solution of the present system in their common domain of existence.

\section{A non-strict hyperbolic system with arbitrary lapse and shift}

LEMMA. The following combination of derivatives of components of the Ricci tensor of an arbitrary spacetime is a quasi diagonal hyperbolic operator for the extrinsic curvature of the space slices:

$$
\Lambda_{i j} \equiv \hat{\partial}_{0} \hat{\partial}_{0} R_{i j}-\hat{\partial}_{0} \bar{\nabla}_{(i} R_{j) 0}+\bar{\nabla}_{j} \partial_{i} R_{00}
$$

Pro of. A straightforward computation using previous results shows that

$$
\begin{aligned}
\Lambda_{i j} \equiv & \hat{\partial}_{0} \square K_{i j}+\hat{\partial}_{0} \hat{\partial}_{0}\left(H K_{i j}-2 K_{i m} K_{j}^{m}\right)-\hat{\partial}_{0} \hat{\partial}_{0}\left(N^{-1} \bar{\nabla}_{j} \partial_{i} N\right)+ \\
& \hat{\partial}_{0}\left(-\bar{\nabla}_{(i}\left(K_{j) h} \partial^{h} N\right)-2 N \bar{R}_{i j m}^{h} K_{h}^{m}-N \bar{R}_{m(i} K_{j)}^{m}+H \bar{\nabla}_{j} \partial_{i} N\right)+ \\
& \bar{\nabla}_{j} \partial_{i}\left(N \bar{\Delta} N-N^{2} K . K\right)+\mathcal{C}_{i j}
\end{aligned}
$$

with

$$
\square K_{i j} \equiv-\hat{\partial}_{0}\left(N^{-1} \hat{\partial}_{0} K_{i j}\right)+\bar{\nabla}^{h} \bar{\nabla}_{h}\left(N K_{i j}\right), \quad \bar{\Delta}=\bar{\nabla}_{h} \bar{\nabla}^{h}
$$

and

$$
\mathcal{C}_{i j} \equiv \bar{\nabla}_{j} \partial_{i}\left(N \partial_{0} H\right)-\hat{\partial}_{0}\left(N \bar{\nabla}_{j} \partial_{i} H\right)
$$

We see that $\mathcal{C}_{i j}$ contains terms of at most second order in $K$ (and also in $N$ ) and first order in $\bar{g}$ (replace $\hat{\partial}_{0} g_{i j}$ by $-2 N K_{i j}$ ).

The identity given above shows that for a solution of the Einstein equations the extrinsic curvature $K$ satisfies, for any choice of lapse and shift, a third order differential system which is quasi diagonal with principal part the hyperbolic operator $\hat{\partial}_{0} \square$. The other unknown $\bar{g}$ appears at second order except for terms appearing through $\bar{\nabla}_{j} \partial_{i} \bar{\Delta} N$.

The system for $\bar{g}$ and $K$ is not hyperbolic in the usual sense of Leray because of the third order space derivatives of $\bar{g}$ appearing in $\bar{\nabla}_{j} \partial_{i} \bar{\Delta} N$.

THEOREM 3. The system for $\bar{g}, K$ is equivalent to a system hyperbolic non-strict in the sense of Leray-Ohya (see [17]) with local existence of solutions in Gevrey classes and domain of dependence determined by the light cone.

P r o of. Replace in the equation $K$ by $-(2 N)^{-1} \hat{\partial}_{0} \bar{g}$ : this gives a quasi diagonal system for $\bar{g}$, but with multiplicity 2 for $\partial_{0}$ in the principal operator.

The system for $\bar{g}, K$ can be turned into a hyperbolic system by a gauge choice (or redefinition of $N$ ) leading either to an elliptic or to a hyperbolic equation for $N$ containing an arbitrary function. Here, we sketch a forthcoming more detailed treatment (see [3]). 
1. We give arbitrarily a constant $c$, taken to be zero in the asymptotically flat case and strictly positive if $M$ is compact. We choose arbitrarily a smooth function $f$ on $M \times \Re$, with appropriate asymptotic behavior if $M$ is euclidean at infinity and $f \leq 0$ and $f \not \equiv 0$ on each $M_{t}$ if they are compact. We require $N$ to satisfy on each $M_{t}$ the elliptic equation depending on $\bar{g}$

$$
\bar{\Delta} N-c N=f
$$

and we determine $N_{0}=\partial_{0} N$ and $N_{00}=\partial_{00}^{2} N$ from the elliptic equations obtained by differentiating the equation for $N$ and replacing $\hat{\partial}_{0} \bar{g}$ by $-2 N K$. It can be shown that the mixed hyperbolic and elliptic system obtained for $\bar{g}, K, N, N_{0}, N_{00}$ is well posed, in appropriate functional spaces.

2. We choose arbitrarily a smooth function $f$ on $M \times \Re$ and require $N$ to satisfy the wave equation.

$$
N^{-2} \partial_{0} \partial_{0} N-\bar{\Delta} N=f
$$

We use this wave equation to reduce the term $\partial_{00}^{2}\left(N^{-1} \bar{\nabla}_{j} \partial_{i} N\right)$ in $\Lambda_{i j}$. It can be shown that the system for $\bar{g}, K, N, N_{0}$ is hyperbolic, hence well posed in local Sobolev spaces, if $N_{0}$ is determined by the hyperbolic equation obtained by differentiating the wave equation satisfied by $N$.

\section{References}

[1] A. Abrahams, A. Anderson, Y. Choquet-Bruhat and J. W. York, Einstein and Yang-Mills theories in hyperbolic form without gauge fixing, Phys. Rev. Letters 75 (1995), $3377-3381$

[2] A. Abrahams, A. Anderson, Y. Choquet-Bruhat and J. W. York, Geometrical hyperbolic systems for general relativity and gauge theories, submitted to Class. Quantum Grav., gr-qc/9605014.

[3] A. Abrahams, A. Anderson, Y. Choquet-Bruhat and J. W. York, A non-strictly hyperbolic system for the Einstein equations with arbitrary lapse and shift, submitted to C.R. Acad. Sci. Paris A.

[4] L. Bel, C.R. Acad. Sci. Paris 246 (1958), 3105.

[5] C. Bona, J. Masso, E. Seidel and J. Stela, A new formalism for numerical relativity, Phys. Rev. Letters 75 (1995), 600-603.

[6] Y. Choquet (Foures)-Bruhat, Sur L'Intégration des Équations de la Relativité Générale, J. Rat. Mechanics and Anal. 5 (1956), 951-966.

[7] Y. Choquet-Bruhat and D. Christodoulou, Elliptic systems in $H_{s, \delta}$ spaces on manifolds which are Euclidean at infinity,Acta. Math. 146 (1981), 129-150.

[8] Y. Choquet-Bruhat and T. Ruggeri, Hyperbolicity of the $3+1$ system of Einstein equations, Commun. Math. Phys. 89 (1983), 269-275.

[9] Y. Choquet-Bruhat and J. W. York, The Cauchy problem in: General Relativity and Gravitation, A. Held (ed.), Plenum, New York, 1980, 99-172.

[10] Y. Choquet-Bruhat and J. W. York, Geometrical well posed systems for the Einstein equations, C.R. Acad. Sci. Paris 321 (1995), Série I, 1089-1095. 
[11] Y. Choquet-Bruhat and J. W. York, Mixed Elliptic and Hyperbolic Systems for the Einstein Equations, in: Gravitation, Electromagnetism and Geometric Structures, G. Ferrarese (ed.) Pythagora Editrice, Bologna, Italy, 1996, 55-73.

[12] D. Christodoulou and S. Klainerman, The Global Nonlinear Stability of the Minkowski Space, Princeton University Press, Princeton, 1993.

[13] H. Friedrich, Hyperbolic reductions for Einstein's equations, Class. Quantum Grav. 13 (1996), 1451-1459.

[14] S. Frittelli and O. Reula, On the Newtonian limit of general relativity, Commun. Math. Phys. 166 (1994), 221-235.

[15] C. Lanczos, A remarkable property of the Riemann-Christoffel tensor in four dimensions, Ann. of Math. 39 (1938), 842-850.

[16] J. Leray, Hyperbolic Differential Equations, Institute for Advanced Study, Princeton, 1952.

[17] J. Leray and Y. Ohya, Équations et systèmes non-linéaires, hyperboliques non-stricts, Math. Ann. 170 (1967), 167-205.

[18] A. Lichnerowicz, Problèmes globaux en Mécanique Relativiste, Hermann, Paris, 1939.

[19] J. W. York, Kinematics and dynamics of general relativity, in: Sources of Gravitaional Radiation, L. Smarr (ed.), Cambridge University Press, Cambridge, 1979, 83-126.

[20] J. W. York, Bel-Robinson Gravitational Superenergy and Flatness, in: Gravitation and Geometry, W. Rindler and A. Trautman (eds.), Bibliopolis, Naples, Italy, 1987, 497-505. 